Curr Opin Neurobiol. 2009 December ; 19(6): 654-660. doi:10.1016/j.conb.2009.10.004.

\title{
Neurobiology of song learning
}

\author{
Richard Mooney \\ Department of Neurobiology, Box 3209, Duke University Medical Center, Durham, NC 27710, \\ United States
}

\begin{abstract}
Birdsong is a culturally transmitted behavior that depends on a juvenile songbird's ability to imitate the song of an adult tutor. Neurobiological studies of birdsong can reveal how a complex form of imitative learning, which bears strong parallels to human speech learning, can be understood at the level of underlying circuit, cellular, and synaptic mechanisms. This review focuses on recent studies that illuminate the neurobiological mechanisms for singing and song learning.
\end{abstract}

\section{Introduction to song learning}

Juvenile songbirds learn to sing during a sensitive period comprising two sequential phases — sensory and sensorimotor learning — both dependent on auditory experience [1,2]. During sensory learning, the juvenile listens to and memorizes one or more tutor songs. In the ensuing phase of sensorimotor learning, which may involve tens or even hundreds of thousands of song repetitions over many weeks, the juvenile uses auditory feedback to match its song to the memorized model. Two hallmarks of sensorimotor learning are high levels of acute (bout to bout) song variability and slower changes (i.e. plasticity) that render the juvenile's song increasingly similar to the memorized tutor song $[3,4]$. Influenced by reinforcement learning theory, a popular though untested idea is that song variability is necessary to these slower adaptive changes. Sensorimotor learning ends with crystallization, wherein the song becomes highly stereotyped and less dependent on auditory feedback $[5,6]$. In the adults of some species, including zebra and Society finches, song slowly deteriorates following deafening or exposure to distorted auditory feedback (DAF), indicating an ongoing role for feedback in song maintenance as well as an ongoing capacity for vocal plasticity [7-9].

In addition to a potential role in song learning, variability is important to song's communication function. The song of an adult male zebra finch directed to a female is slightly less variable than the undirected songs it sings in isolation [10,11]. Interestingly, female finches are more attracted to directed songs, even those produced by unfamiliar males, indicating they are sensitive to these very slight differences [12]. Practically, social effects on song afford neuroscientists a useful tool for probing neural mechanisms driving song variability. 
Before delving into the neurobiology of singing and song learning, it is useful to consider what the juvenile songbird's brain must accomplish to successfully copy a tutor's song. One is to precisely control the peripheral vocal-respiratory apparatus. Another is to compare auditory feedback to the tutor song memory to detect vocal 'errors.' Finally, when errors are detected, the brain must generate an instructive signal that modifies song motor commands, subsequently minimizing these errors. A spate of recent studies provide an increasingly detailed description of the central mechanisms for singing and generating song variability, and have also begun to provide important clues about the link between variability and vocal plasticity. Moreover, recent advances have identified central substrates for auditory feedback and possibly for tutor song memories. These findings set the stage for understanding how these auditory representations are compared and used to adaptively modify song during sensorimotor learning.

\section{Introduction to neural circuits for song}

The songbird's brain contains a system of interconnected brain nuclei specialized for singing and song learning (i.e. the song system; Figure 1) [13,14]. The song system comprises two major parts, a song motor pathway (SMP), which spans from the telencephalon to the brainstem vocal-respiratory network, and an anterior forebrain pathway (AFP) that traverses the telencephalon, striatum, and thalamus, and which resembles mammalian cortical-basal ganglia pathways [15]. Chronic recordings in singing birds reveal that SMP and AFP neurons exhibit time-locked singing-related activity that can increase before vocalization and that can persist following deafening, consistent with a motor origin [16,17]. Lesions in the SMP abolish or permanently disrupt singing, indicating this pathway serves an essential song motor role [18]. Juvenile finches with lesions to the AFP output nucleus, LMAN, continue to sing, but their songs become less variable and appear to crystallize prematurely $[19,20]$. Adult zebra finches with LMAN lesions produce crystallized songs normally, but their songs do not display context-dependent changes in variability and do not deteriorate following deafening $[19,21]$. These findings suggest that the AFP functions similarly in juvenile and adult birds to enable both song variability and audition-dependent song plasticity.

Two anatomical features of the song system are especially noteworthy (Figure 1A). One is that the SMP and the AFP receive input from different populations of projection neurons in the telencephalic nucleus $\mathrm{HVC}$ ( $\mathrm{HVC}_{\mathrm{RA}}$ and $\mathrm{HVC}_{\mathrm{X}}$ neurons), making it a common source for the singing-related activity that can be detected in both pathways. The other feature is that RA, which lies immediately downstream of HVC in the SMP, is the sole target of the AFP output nucleus LMAN. This arrangement suggests that RA is the site where the AFP influences song variability and plasticity. These anatomical features motivate intense interest in analyzing singing-related activity in HVC and RA and understanding how song motor activity in RA is modulated by LMAN activity.

\section{Song motor codes and central mechanisms for generating song variability}

Current evidence places HVC at the apex of a song motor patterning network. Even though HVC receives input from several other song nuclei, HVC is the song nucleus most removed 
from the vocal periphery where neurons display time-locked singing-related activity and where lesions permanently disrupt song. What is the nature of the song motor code transmitted by HVC? A landmark study in singing zebra finches showed that individual $\mathrm{HVC}_{\mathrm{RA}}$ neurons fire only once per entire motif (a poly-syllabic phrase that is the largest unit of song learning, $\sim 0.5-1 \mathrm{~s}$ in duration), then only very briefly ( $\sim 10 \mathrm{~ms})$ and at very high frequency $(\sim 400 \mathrm{~Hz})$ [22]. In a single bird, different $\mathrm{HVC}_{\mathrm{RA}}$ neurons burst at different times during the motif and some cells even fired during silent gaps between syllables.

One model fostered by these findings posits that the entire ensemble of $\sim 40000 \mathrm{HVC}_{\mathrm{RA}}$ neurons acts as a high frequency $(\sim 100 \mathrm{~Hz})$ clock to encode song tempo. Although this idea is attractive, the electrophysiological data are insufficient to resolve whether this signal originates in $\mathrm{HVC}$ and whether it functions specifically to encode temporal features of song. Importantly, a recent study found that song tempo slowed when HVC was focally cooled with a small Peltier device, providing answers to these questions [23]. Rather remarkably, this effect was uniform from the millisecond timescale of individual notes to the second-long scale of the entire motif, including the silent gaps between syllables. This effect also was specific to timing with other song features, such as frequency and amplitude, remaining largely unchanged. Because focal cooling of HVC should affect the biophysical properties of the HVC microcircuit without altering the timing of activity in HVC's afferents, these findings suggest that $\mathrm{HVC}$ plays a major role in generating timing signals for song. Nonetheless, pathways from brainstem SMP components to HVC (Figure 1B) are likely to trigger initiation or termination of syllables, because electrical stimulation in the brainstem can truncate and restart the motif [24] and because intersyllable durations are more variable than syllable durations [25]. An important future goal will be to define the cellular and circuit mechanisms that generate and propagate bursting activity in $\mathrm{HVC}_{\mathrm{RA}}$ neurons, and to understand how this activity is initiated and terminated to pattern song.

In contrast to $\mathrm{HVC}_{\mathrm{RA}}$ neurons, individual RA neurons burst up to 10 times per motif [26], raising the possibility that a timing signal from $\mathrm{HVC}$ is transformed in RA into a signal more specifically correlated with acoustical features of the bird's song. However, the mean population activity in RA and mean spectral features of song are on average uncorrelated, indicating that different ensembles of RA neurons can contribute to similar sounds at different times in the motif [26]. Nonetheless, a recent study found that trial to trial variability in individual RA neurons can predict a significant portion of trial to trial acoustic variability in the bird's song [27]. Therefore, a plausible idea is that factors that drive variations in the firing patterns of ensembles of RA neurons could drive variable performance, a major attribute of sensorimotor learning.

In fact, numerous lines of evidence indicate that LMAN drives song variability. First, in juvenile birds engaged in sensorimotor learning, inactivating LMAN sharply reduces song variability [28]. Second, in adult male zebra finches, LMAN lesions abolish contextdependent changes in song variability, an effect attributable to reduced variability of undirected songs [21]. Third, singing-related activity in LMAN changes with social context [11], with LMAN neurons displaying more variable spike timing and more frequent bursting during undirected singing $[29,30]$. Fourth, spike timing variability and 'burstiness' in LMAN are highest during sensorimotor learning, when song variability also is most 
pronounced [28]. Fifth, the electrical stimulation in LMAN during singing can alter the acoustical features of the stimulated syllable [31]. Together, these findings suggest that variable LMAN firing patterns, especially variable bursting activities of LMAN neurons, drive song variability.

Current evidence also suggests a fast synaptic mechanism by which LMAN could affect RA firing patterns to modulate song variability. Terminals of LMAN and HVC axons commingle on dendrites of RA projections neurons, where LMAN synapses activate NMDA receptors and HVC synapses activate both AMPA and NMDA receptors [32-34]. When $\mathrm{HVC}_{\mathrm{RA}}$ and LMAN neurons fire together during singing, voltage-dependent blockade of NMDA receptors could be relieved, permitting LMAN inputs to contribute to postsynaptic depolarization of RA neurons. This arrangement may enable variably firing LMAN neurons to add 'noise' in a conditional manner to the motor signals from HVC, causing the singingrelated firing patterns of RA neurons - and thus song — to become more variable. In support of this view, studies in juvenile zebra finches show that inactivating LMAN reduces RA spike time variability and blocking NMDA receptors in RA immediately reduces bout to bout song variability $[28,35]$.

One important goal is to clarify whether and how variability is linked to the slower adaptive changes that occur to song during sensorimotor learning. Although the findings that LMAN lesions reduce song variability and plasticity hint that these processes could be linked, a causal role for variability in sensorimotor learning remains speculative. Another important goal will be to determine the source and nature of instructive signals for song learning. More broadly, the finding that LMAN lesions prevent various forms of juvenile and adult song plasticity, including those triggered by disrupting auditory feedback, is consistent with the idea that LMAN transmits an instructive signal. Alternatively, LMAN could function permissively, enabling instructive signals arising from other sources to modify song motor commands at the level of RA.

Exciting new evidence suggests that LMAN can drive variability in a biased rather than purely random manner, consistent with an instructive role [36]. Crystallized songs of adult finches exhibit small levels of variability, for example in the pitch (i.e. fundamental frequency) of their syllables. When an adult finch is exposed to noise bursts whenever it sings higher pitch variants of a target syllable, it slowly ( hours) shifts the syllable's pitch downward, subsequently reducing the probability that singing will trigger noise playback [37]. The initial expression of this adaptive shift depends on the AFP, because pharmacologically silencing LMAN causes the target syllable's pitch to immediately revert toward its original value [36]. This finding cannot exclude the possibility that LMAN functions permissively to gate a bias signal arising from another source (i.e. HVC). Nonetheless, LMAN neurons exhibit song premotor activity and electrically stimulating subregions of LMAN during singing alters the targeted syllable in a consistent way, suggesting LMAN can provide an instructive bias. A further unresolved issue is whether the mechanisms that enable adaptive pitch shifts in the adult birds are the same as those that enable juvenile sensorimotor learning. Therefore, an important goal will be to determine whether LMAN normally functions during sensorimotor learning to bias song in such a way to minimize the differences between auditory feedback and memorized tutor song. 


\section{The search for central representations of auditory feedback}

Instructive signals for sensorimotor learning must ultimately arise from feedback-dependent performance evaluation. A parsimonious idea is that singing-related auditory feedback is processed by the AFP, enabling this pathway to evaluate performance and instructively modify song motor commands. In fact, recordings made in the AFP of anesthetized birds detect neurons that respond selectively to playback of the bird's own song, fueling speculation that the AFP receives feedback-related information [38].

Despite the attractiveness of this idea, the singing-related activity of LMAN neurons appears to be insensitive to acute exposure to distorted auditory feedback (DAF) [39], even though the chronic exposure to DAF triggers song plasticity. Although LMAN neurons do not convey a real time feedback signal, the auditory selectivity of LMAN neurons in adult finches can shift over days to weeks in response to chronic feedback perturbations, and this auditory plasticity manifests before the onset of vocal plasticity [40]. These findings hint that feedback signals may be processed at earlier stages of the AFP, and the resulting evaluation acts more slowly to influence LMAN activity. Testing this idea requires assessing whether the inputs to the AFP are sensitive to feedback perturbations.

Two major inputs to the earliest stage of the AFP (i.e. area X) are HVC and the midbrain ventral tegmental area (VTA) (Figure 1A). In mammals, certain dopaminergic VTA neurons are implicated in reinforcement learning [41], raising speculation that they could serve a similar function in songbirds to facilitate sensorimotor learning. Interestingly, in adult male zebra finches, singing-related activity of VTA neurons changes with social context [42], raising the possibility that the VTA could influence song variability through its connections with area X. If VTA neurons also receive information evaluating song performance, then they may provide a means of reinforcing certain patterns of variability in the AFP, resulting in a biased (i.e. instructive) variability signal. Therefore, an important goal of future studies will be to determine whether VTA neurons process feedback and/or encode information about vocal errors.

Although a possible role for the VTA in song learning is an intriguing idea, a major source of auditory and singing-related information to the AFP is HVC, making AFP-projecting $\mathrm{HVC}$ neurons (i.e. $\mathrm{HVC}_{\mathrm{X}}$ cells) a logical place to search for feedback signals. However, experiments combining chronic recordings and DAF in both juvenile and adult songbirds indicate that the singing-related activity of $\mathrm{HVC}_{\mathrm{X}}$ neurons is insensitive to acute feedback perturbation $[43,44]$. Apparently, HVC sends an efference copy to the AFP of the song motor commands it transmits to RA. Indeed, $\mathrm{HVC}_{\mathrm{RA}}$ neurons make monosynaptic and disynaptic connections with $\mathrm{HVC}_{\mathrm{X}}$ cells [45], providing a substrate for relaying an efference copy to the AFP.

A recent study sheds further light on the nature of this efference copy. Chronic recordings from $\mathrm{HVC}_{\mathrm{X}}$ cells in both adult swamp sparrows and Society finches show that some of these neurons display similar patterns of activity when the bird sings its song or passively listens to it through a speaker [43]. The precise sensorimotor correspondence displayed by $\mathrm{HVC}_{\mathrm{X}}$ cells is reminiscent of 'mirror neurons' in frontal regions of the monkey cortex, which are 
hypothesized to facilitate mimicry and communication [46,47]. Indeed, as hypothesized for mirror neurons in monkeys, the sensory properties of these $\mathrm{HVC}_{\mathrm{X}}$ mirror neurons are tightly linked to song perception [48]. One interesting idea is that this sensorimotor correspondence enables the motor-related activity of $\mathrm{HVC}_{\mathrm{X}}$ cells to be compared to the actual feedback signal, thus facilitating the detection of vocal errors. If this model is correct, then neurons that receive synaptic input from $\mathrm{HVC}_{\mathrm{X}}$ cells and that also respond to auditory feedback could such comparisons. Alternatively, $\mathrm{HVC}_{\mathrm{X}}$ mirror neurons could be a site of comparison, but in the adult birds in which they were detected, the feedback signals they receive may be overwhelmed by song motor-related signals, an arrangement that may favor song stability. Complete resolution of these issues will require testing feedback sensitivity of $\mathrm{HVC}_{\mathrm{X}}$ cells at the earliest stages of sensorimotor learning, when song is most sensitive to feedback perturbation.

The songbird hears its song only when it sings, so performance evaluation requires neurons that respond in real time to vocalization-related auditory feedback. Two recent studies have identified such neurons $[49,50]$. One of these reported that DAF could slightly and transiently suppress singing-related multiunit activity in HVC of adult Society finches, providing the first clue that auditory feedback may be processed in the song system [51]. Because multiunit recordings in HVC are thought to represent interneuron activity, this finding suggests several circuit mechanisms by which feedback could affect song. First, some $\mathrm{HVC}_{\mathrm{X}}$ cells receive inhibitory synaptic input from interneurons and excitatory input from $\mathrm{HVC}_{\mathrm{RA}}$ cells [45], which could enable $\mathrm{HVC}_{\mathrm{X}}$ cells to compare a real time feedback signal with motor-related signals. Alternatively or additionally, interneurons could compare real and predicted feedback signals, because they receive excitatory input from both extrinsic auditory sources and from $\mathrm{HVC}_{\mathrm{X}}$ cells $[45,52]$. Finally, some HVC interneurons make synaptic connections onto $\mathrm{HVC}_{\mathrm{RA}}$ cells, providing a route via which feedback could directly influence song motor commands [45].

Another recent report described feedback-sensitive neurons in Field L and CLM [50], avian analogs to mammalian primary and secondary auditory cortices, respectively. These neurons could be divided into three functional classes: first, neurons with similar patterns of activity during singing and song playback, and in which singing-related activity was sensitive to DAF; second, neurons showing similar patterns of activity during singing and song playback and that were insensitive to DAF; and third, a rarer type was largely inactive except when singing-related feedback was disrupted. The authors raise the interesting idea that these three cell types reflect components of a circuit that combines a feedback representation with a motor-based predictive signal to generate signals for error correction. To move beyond speculation, however, it will be necessary to determine whether these cell types functionally interact with one another to detect vocal errors and whether the third cell type provides input to the song system, providing a means for it to influence song. One hint that these cells could influence singing is the finding that some neurons in CLM provide an important source of auditory input to HVC [53]. More generally, an important goal will be to assess whether the activity of these or other feedback-sensitive neurons is harnessed for sensorimotor learning. As a first step toward this goal, it would be useful to determine whether disrupting the singing-related activity of feedback-sensitive neurons, perhaps through the use of singing-triggered microstimulation, can trigger song plasticity. 


\section{The search for central representations of tutor song memories}

How and where tutor song memories might be encoded in the juvenile's brain remains a matter of debate. Two lines of evidence have implicated the AFP: first, when NMDA receptors in LMAN of juvenile zebra finches are transiently blocked during tutoring, copying is subsequently impaired [54]; second, recordings in anesthetized zebra finches during sensorimotor learning detect some LMAN neurons that are selective for the tutor's song [55]. Intriguingly, some tutor-selective cells could be detected even in juvenile birds with severed vocal nerves [56]. Because this manipulation makes the bird's song very different from the tutor song, these tutor-selective cells may encode a tutor song memory. Nonetheless, the absence of a robust and persistent tutor representation in the song system has motivated an expanded search in regions of the auditory telencephalon, particularly NCM, an area broadly implicated in auditory plasticity [57]. Notably, IEG expression levels in NCM correlate with how well the bird copied the tutor song [58-60] and NCM neuronal firing rates in adult finches habituate more slowly to playback of tutor song than to novel songs, with the slowest habituation rates for those tutor songs copied most accurately [61]. Thus various lines evidence support localization either within or outside the song system.

Two recent findings may further inform this debate $[62,63]$. First, reversibly blocking mitogen-activated protein kinase/extracellular signal-regulated kinase (MEK) signaling in NCM in juvenile zebra finches during, but not after tutoring, was found to impair copying [62]. Although the electrophysiological consequences in NCM of such treatment were not characterized, studies in other systems hint that interfering with MEK signaling selectively impacts synaptic plasticity important to memory formation, rather than interfering with basal synaptic transmission [64]. Second, chronic recordings of sleep-related activity in the RA of juvenile finches revealed a sharp increase in bursting activity in the first night after their exposure to a tutor song [63]. Intriguingly, bursting in RA increased before vocal evidence of imitation and RA neurons in different birds exposed to the same tutor song displayed similar patterns of bursting activity. However, the emergence of bursting activity could be delayed using muting or noise-masking, suggesting that this functional change depends on an instructive interaction between the tutor memory and the song motor network. These findings suggest a scenario where the effects of tutor song experience propagate rapidly from the auditory telencephalon into the song system as soon as feedback is compared to the tutor song memory.

\section{References}

1. Catchpole, C.; Slater, P. Bird Song: Biological Themes and Variations. Cambridge, UK: Cambridge University Press; 1995.

2. Marler P. Song learning: the interface between behaviour and neuroethology. Philos Trans R Soc Lond B: Biol Sci. 1990; 329:109-114. [PubMed: 1978357]

3. Deregnaucourt S, Mitra PP, Feher O, Pytte C, Tchernichovski O. How sleep affects the developmental learning of bird song. Nature. 2005; 433:710-716. [PubMed: 15716944]

4. Marler P, Peters S. Structural changes in song ontogeny in the Swamp Sparrow Melospiza georgiana. Auk. 1982; 99:446-458.

5. Konishi M. The role of auditory feedback in the control of vocalization in the white-crowned sparrow. Z Tierpsychol. 1965; 22:770-783. [PubMed: 5874921] 
6. Price R. Developmental determinants of structure in zebra finch song. Comp Phys Psychol. 1979:268-277.

7. Leonardo A, Konishi M. Decrystallization of adult birdsong by pertubation of auditory feedback. Nature. 1999; 399:466-470. [PubMed: 10365958]

8. Nordeen KW, Nordeen EJ. Auditory feedback is necessary for the maintenance of stereotyped song in adult zebra finches. Behav Neural Biol. 1992; 57:58-66. [PubMed: 1567334]

9. Woolley SM, Rubel EW. Bengalese finches Lonchura Striata domestica depend upon auditory feedback for the maintenance of adult song. J Neurosci. 1997; 17:6380-6390. [PubMed: 9236246]

10. Sossinka R, Bohner J. Song types in the zebra finch Poephila guttata castanotis. Z Tierpsychol. 1980; 53:123-132.

11. Jarvis ED, Scharff C, Grossman MR, Ramos JA, Nottebohm F. For whom the bird sings: context dependent gene expression. Neuron. 1998; 21:775-788. [PubMed: 9808464]

12. Woolley SC, Doupe AJ. Social context-induced song variation affects female behavior and gene expression. PLoS Biol. 2008; 6:e62. [PubMed: 18351801]

13. Nottebohm F, Alvarez-Buylla A, Cynx J, Kirn J, Ling CY, Nottebohm M, Suter R, Tolles A, Williams H. Song learning in birds: the relation between perception and production. Philos Trans R Soc Lond B: Biol Sci. 1990; 329:115-124. [PubMed: 1978358]

14. Nottebohm F, Kelley DB, Paton JA. Connections of vocal control nuclei in the canary telencephalon. J Comp Neurol. 1982; 207:344-357. [PubMed: 7119147]

15. Doupe AJ, Perkel DJ, Reiner A, Stern EA. Birdbrains could teach basal ganglia research a new song. Trends Neurosci. 2005; 28:353-363. [PubMed: 15935486]

16. McCasland JS, Konishi M. Interaction between auditory and motor activities in an avian song control nucleus. Proc Natl Acad Sci U S A. 1981; 78:7815-7819. [PubMed: 6950421]

17. Hessler NA, Doupe AJ. Singing-related neural activity in a dorsal forebrain-basal ganglia circuit of adult zebra finches. J Neurosci. 1999; 19:10461. [PubMed: 10575043]

18. Nottebohm F, Stokes TM, Leonard CM. Central control of song in the canary, Serinus canarius. J Comp Neurol. 1976; 165:457-486. [PubMed: 1262540]

19. Bottjer SW, Miesner EA, Arnold AP. Forebrain lesions disrupt development but not maintenance of song in passerine birds. Science. 1984; 224:901-903. [PubMed: 6719123]

20. Scharff C, Nottebohm F. A comparative study of the behavioral deficits following lesions of various parts of the zebra finch song system: implications for vocal learning. J Neurosci. 1991; 11:2896-2913. [PubMed: 1880555]

21. Kao MH, Brainard MS. Lesions of an avian basal ganglia circuit prevent context-dependent changes to song variability. J Neurophysiol. 2006; 96:1441-1455. [PubMed: 16723412]

22. Hahnloser R, Kozhevnikov A, Fee M. An ultra-sparse code underlies the generation of neural sequences in a songbird. Nature. 2002; 419:65-70. [PubMed: 12214232]

23. Long MA, Fee MS. Using temperature to analyse temporal dynamics in the songbird motor pathway. Nature. 2008; 456:189-194. [PubMed: 19005546]

24. Ashmore RC, Wild JM, Schmidt MF. Brainstem and forebrain contributions to the generation of learned motor behaviors for song. J Neurosci. 2005; 25:8543-8554. [PubMed: 16162936]

25. Glaze CM, Troyer TW. Temporal structure in zebra finch song: implications for motor coding. $\mathbf{J}$ Neurosci. 2006; 26:991-1005. [PubMed: 16421319]

26. Leonardo A, Fee MS. Ensemble coding of vocal control in birdsong. J Neurosci. 2005; 25:652661. [PubMed: 15659602]

27. Sober SJ, Wohlgemuth MJ, Brainard MS. Central contributions to acoustic variation in birdsong. J Neurosci. 2008; 28:10370-10379. [PubMed: 18842896]

28. Olveczky BP, Andalman AS, Fee MS. Vocal experimentation in the juvenile songbird requires a basal ganglia circuit. PLoS Biol. 2005; 3:e153. [PubMed: 15826219]

29. Hessler NA, Doupe AJ. Social context modulates singing-related neural activity in the songbird forebrain. Nat Neurosci. 1999; 2:209-211. [PubMed: 10195211]

30. Kao MH, Wright BD, Doupe AJ. Neurons in a forebrain nucleus required for vocal plasticity rapidly switch between precise firing and variable bursting depending on social context. $\mathrm{J}$ Neurosci. 2008; 28:13232-13247. [PubMed: 19052215] 
31. Kao MH, Doupe AJ, Brainard MS. Contributions of an avian basal ganglia-forebrain circuit to realtime modulation of song. Nature. 2005; 433:638-643. [PubMed: 15703748]

32. Canady RA, Burd GD, DeVoogd TJ, Nottebohm F. Effect of testosterone on input received by an identified neuron type of the canary song system: a Golgi/electron microscopy/ degeneration study. J Neurosci. 1988; 8:3770-3784. [PubMed: 2461435]

33. Mooney R. Synaptic basis for developmental plasticity in a birdsong nucleus. J Neurosci. 1992; 12:2464-2477. [PubMed: 1351935]

34. Stark LL, Perkel DJ. Two-stage, input-specific synaptic maturation in a nucleus essential for vocal production in the zebra finch. J Neurosci. 1999; 19:9107-9116. [PubMed: 10516328]

35. Olveczky B, Fee M. Effects of LMAN on the premotor representation of song in juvenile songbirds. Soc Neurosci. 2006 [Epub 2006].

36. Andalman AS, Fee MS. A basal ganglia-forebrain circuit in the songbird biases motor output to avoid vocal errors. Proc Natl Acad Sci U S A. 2009

37. Tumer EC, Brainard MS. Performance variability enables adaptive plasticity of 'crystallized' adult birdsong. Nature. 2007; 450:1240-1244. [PubMed: 18097411]

38. Doupe AJ, Konishi M. Song-selective auditory circuits in the vocal control system of the zebra finch. Proc Natl Acad Sci U S A. 1991; 88:11339-11343. [PubMed: 1763048]

39. Leonardo A. Experimental test of the birdsong error-correction model. Proc Natl Acad Sci U S A. 2004; 101:16935-16940. [PubMed: 15557558]

40. Roy A, Mooney R. Auditory plasticity in a basal ganglia-forebrain pathway during decrystallization of adult birdsong. J Neurosci. 2007; 27:6374-6387. [PubMed: 17567798]

41. Schultz W. Getting formal with dopamine and reward. Neuron. 2002; 36:241-263. [PubMed: 12383780]

42. Yanagihara S, Hessler NA. Modulation of singing-related activity in the songbird ventral tegmental area by social context. Eur J Neurosci. 2006; 24:3619-3627. [PubMed: 17229110]

43. Prather JF, Peters S, Nowicki S, Mooney R. Precise auditory-vocal mirroring in neurons for learned vocal communication. Nature. 2008; 451:305-310. [PubMed: 18202651]

44. Kozhevnikov AA, Fee MS. Singing-related activity of identified HVC neurons in the zebra finch. J Neurophysiol. 2007; 97:4271-4283. [PubMed: 17182906]

45. Mooney R, Prather JF. The HVC microcircuit: the synaptic basis for interactions between song motor and vocal plasticity pathways. J Neurosci. 2005; 25:1952-1964. [PubMed: 15728835]

46. Rizzolatti G. The mirror neuron system and its function in humans. Anat Embryol (Berl). 2005; 210:419-421. [PubMed: 16222545]

47. Rizzolatti G, Craighero L. The mirror-neuron system. Annu Rev Neurosci. 2004; 27:169-192. [PubMed: 15217330]

48. Prather JF, Nowicki S, Anderson RC, Peters S, Mooney R. Neural correlates of categorical perception in learned vocal communication. Nat Neurosci. 2009; 12:221-228. [PubMed: 19136972]

49. Sakata JT, Brainard MS. Online contributions of auditory feedback to neural activity in avian song control circuitry. J Neurosci. 2008; 28:11378-11390. [PubMed: 18971480]

50. Keller GB, Hahnloser RH. Neural processing of auditory feedback during vocal practice in a songbird. Nature. 2009; 457:187-190. [PubMed: 19005471]

51. Sakata JT, Brainard MS. Real-time contributions of auditory feedback to avian vocal motor control. J Neurosci. 2006; 26:9619-9628. [PubMed: 16988032]

52. Rosen MJ, Mooney R. Synaptic interactions underlying song-selectivity in the avian nucleus HVC revealed by dual intracellular recordings. J Neurophysiol. 2006; 95:1158-1175. [PubMed: 16424457]

53. Bauer EE, Coleman MJ, Roberts TF, Roy A, Prather JF, Mooney R. A synaptic basis for auditoryvocal integration in the songbird. J Neurosci. 2008; 28:1509-1522. [PubMed: 18256272]

54. Basham ME, Nordeen EJ, Nordeen KW. Sensory acquisition in zebra finches requires activation of NMDA receptors in the anterior forebrain. Soc Neurosci. 1995; 21:959.

55. Solis MM, Doupe AJ. Anterior forebrain neurons develop selectivity by an intermediate stage of birdsong learning. J Neurosci. 1997; 17:6447-6462. [PubMed: 9236252] 
56. Solis MM, Doupe AJ. Contributions of tutor and bird's own song experience to neural selectivity in the songbird anterior forebrain. J Neurosci. 1999; 19:4559-458. [PubMed: 10341255]

57. Bolhuis JJ, Gahr M. Neural mechanisms of birdsong memory. Nat Rev Neurosci. 2006; 7:347-357. [PubMed: 16760915]

58. Bolhuis JJ, Zijlstra GG, den Boer-Visser AM, Van Der Zee EA. Localized neuronal activation in the zebra finch brain is related to the strength of song learning. Proc Natl Acad Sci U S A. 2000; 97:2282-2285. [PubMed: 10681421]

59. Bolhuis JJ, Hetebrij E, Den Boer-Visser AM, De Groot JH, Zijlstra GG. Localized immediate early gene expression related to the strength of song learning in socially reared zebra finches. Eur $\mathbf{J}$ Neurosci. 2001; 13:2165-2170. [PubMed: 11422458]

60. Terpstra NJ, Bolhuis JJ, den Boer-Visser AM. An analysis of the neural representation of birdsong memory. J Neurosci. 2004; 24:4971-4977. [PubMed: 15163689]

61. Phan ML, Pytte CL, Vicario DS. Early auditory experience generates long-lasting memories that may subserve vocal learning in songbirds. Proc Natl Acad Sci U S A. 2006; 103:1088-1093. [PubMed: 16418265]

62. London SE, Clayton DF. Functional identification of sensory mechanisms required for developmental song learning. Nat Neurosci. 2008; 11:579-586. [PubMed: 18391944]

63. Shank SS, Margoliash D. Sleep and sensorimotor integration during early vocal learning in a songbird. Nature. 2008

64. Sweatt JD. Mitogen-activated protein kinases in synaptic plasticity and memory. Curr Opin Neurobiol. 2004; 14:311-317. [PubMed: 15194111] 


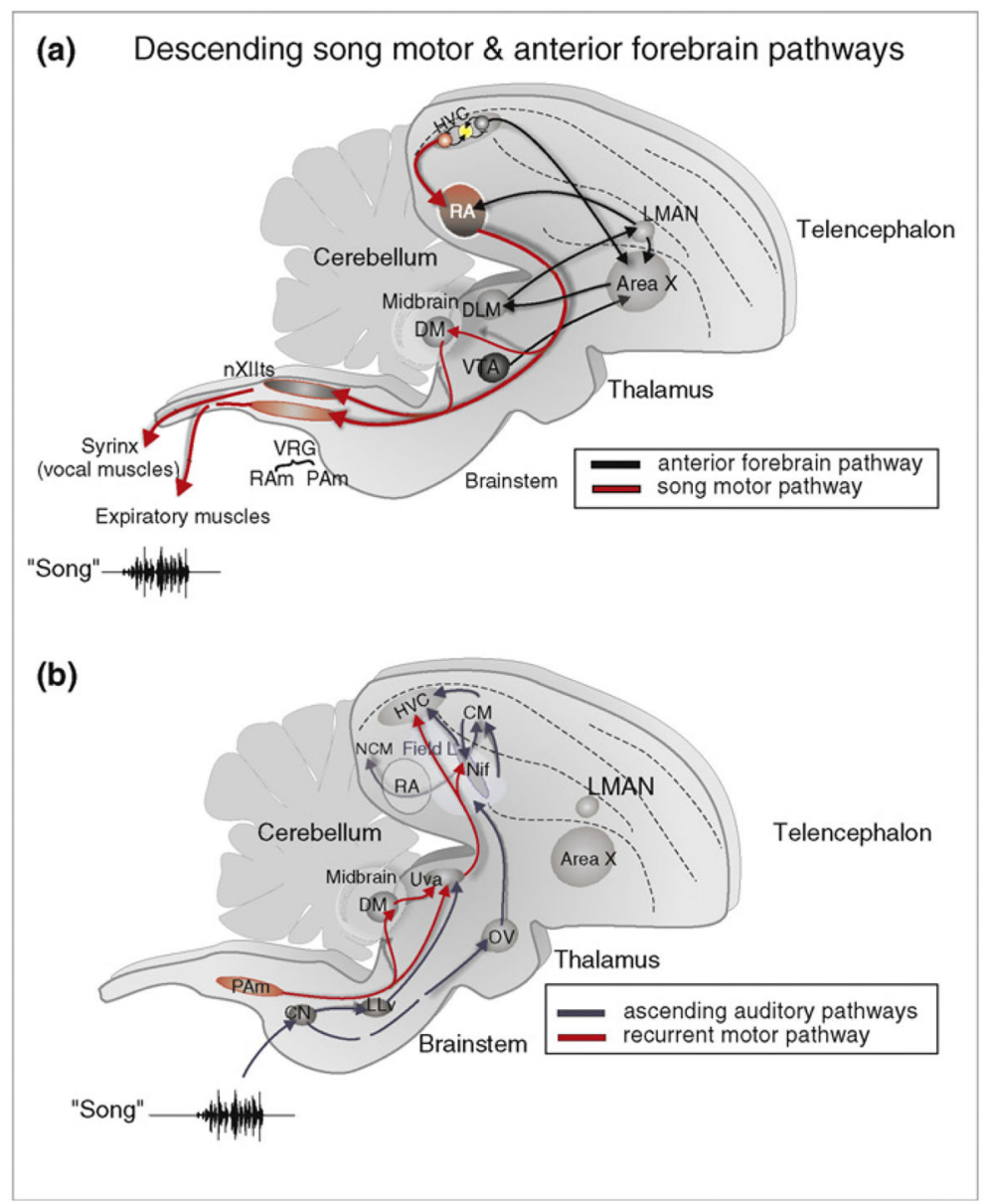

Figure 1.

The song system and ascending sensory and sensorimotor pathways. (A) The song motor pathway (SMP; red) and the anterior forebrain pathway (AFP; black) shown in a parasagittal view. The SMP arises from neurons in $\mathrm{HVC}\left(\mathrm{HVC}_{\mathrm{RA}}\right.$ neurons; $\mathrm{HVC}$ is used as a proper name) that project directly to the robust nucleus of the arcopallium (RA). RA axons terminate on motorneurons in the tracheosyringeal portion of the hypoglossal motor nucleus (XIIts) and on respiratory premotor neurons in the ventral respiratory group (VRG). The VRG comprises the nucleus retroambigualis (RAm), which controls expiration, and the nucleus parambigualis (PAm), which controls inspiration. RA axons also terminate in the dorsomedial intercollicular nucleus (DM) in the midbrain, which plays a role in call generation in birds. The AFP receives input from a population of $\mathrm{HVC}$ neurons $\left(\mathrm{HVC}_{\mathrm{X}}\right.$ neurons), and from the midbrain ventral tegmental area (VTA). Area X provides inhibitory input to the medial nucleus of the dorsolateral thalamus (DLM), which in turn provides excitatory drive to the lateral portion of the magnocellular nucleus of the anterior nidopallium (LMAN). LMAN innervates area $\mathrm{X}$ and also the same RA neurons that receive input from HVC. Thus, the SMP and AFP arise from distinct pools of HVC projections neurons and innervate the same RA song premotor neurons. (B) Pathways that convey auditory and recurrent song motor information to HVC. Auditory information (blue arrows) is relayed from the cochlear nucleus $(\mathrm{CN})$ indirectly to $\mathrm{HVC}$ through two pathways. One 
pathway includes the ventral portion of the lateral lemniscus (LLv) and the thalamic nucleus Uvaformis (Uva). Another pathway includes an indirect pathway (broken line) through the auditory hindbrain and midbrain (not shown) to the thalamic nucleus ovoidalis (Ov); axons from Ov terminate in the telencephalic area Field L, an analog of the mammalian auditory cortex. From Field L, activity is relayed through an interconnected network comprising the caudal medial nidopallium (NCM) and the caudal mesopallium (CM), which in turn projects directly to HVC and indirectly to HVC through the nucleus interfacialis (NIf). 